\title{
Drug Susceptibility pattern of Organisms Isolated During Acute Exacerbation of Chronic Obstructive Pulmonary Disease in a Tertiary Level Hospital of Nepal
}

\author{
Sanjeet Krishna Shrestha', Raina Chaudhary', Sabita Bhatta', Manan Karki ${ }^{3}$ Brajendra \\ Srivastava ${ }^{4}$, Ushab Rana Mousin ${ }^{4}$, Rabindra Khunjeli, Jyoti Shrestha ${ }^{5}$
}

\begin{abstract}
${ }^{1}$ Department of Internal Medicine, Kathmandu Medical College Teaching Hospital, Kathmandu. ${ }^{2}$ Department of Microbiology, Shree Birendra Hospital, Kathmandu. ${ }^{3}$ Oxford University Clinical Research Unit (Nepal), Patan Hospital, Lalitpur. ${ }^{4}$ Department of Respiratory Medicine, Shree Birendra Hospital, Kathmandu. ${ }^{5}$ Department of Anaesthesiology, Kathmandu Medical College Teaching Hospital, Kathmandu, Nepal.
\end{abstract}

\section{ABSTRACT}

Introduction: Disease process in chronic obstructive lung disease is interrupted by acute exacerbations. Bacterial and viral infections account for majority of cases. Gram positive and Gram negative organism both are associated with exacerbations. Methods: Sputum samples were collected from COPD patients in acute exacerbation, on the day of emergency visit. Drug susceptibility pattern was evaluated for the study population to identify the prevalence of susceptible and resistant organisms. Results: Eighty-nine culture positive sputum samples were processed for drug susceptibility. Gram negative bacilli $(88.76 \%)$ were isolated more than Gram positive cocci (11.24\%). Pseudomonas sp., Acinetobacter sp., Klebseilla sp. and E. coli were the most common Gram negative bacilli. Multi-drug resistance status was identified in higher percents in Acinetobacter sp. (81.25\%), Pseudomonas sp. (62.5\%) and Klebseilla sp. (46.6\%). Aminoglycosides and Quinolones showed good sensitivity to GNBs. However, Carbapenems were found to be the most effective agents against these organisms. Conclusion: Gram negative infection is common in COPD. Multi drug resistant pathogens are increasingly associated with acute exacerbations. Routinely used antibiotics are becoming less effective.

Keywords: chronic obstructive pulmonary disease; drug resistance; pseudomonas aeruginosa; acinetobacter

\section{INTRODUCTION}

Chronic Obstructive Pulmonary Disease (COPD), a chronic yet life threatening lung disease, is often interrupted by acute exacerbations (AE). ${ }^{1}$ COPD exacerbations are mostly due to bacterial and viral infections, pollution and cold weather. ${ }^{2,} 3$ Bacterial exacerbations account for about $40-50 \%$ of the cases. ${ }^{3,} 4$ It is observed that there is MJSBH Vol 15 Issue 1 Jan- Jun 2016 colonization of pathogenic bacteria in lower respiratory tract which increases during acute exacerbation. ${ }^{5}$

The prevalent pathogens for infective exacerbations are Pseudomonas aeruginosa,

Correspondence: Department of Internal Medicine, Pulmonary Medicine Division, Kathmandu Medical College Teaching Hospita l, Kathmandu. Emai 1: shrestha.sanjeet@gmail.com 
Haemophilus influenzae, Klebsiella pneumoniae, Moraxella catarrhalis, Streptococcus pneumoniae and Staphylococcus aureus. ${ }^{4,6,7}$ During the last two decades, prevalence of multidrug resistant (MDR) bacteria has increased in all hospitalized patients, including patients with COPD exacerbations. ${ }^{8}$ MDR bacteria were defined as Methicillin-resistant Staphylococcus aureus (MRSA), Ceftazidime or Imipenem resistant Pseudomonas aeruginosa, Acinetobacter baumanii, Stenotrophomonas maltophilia, and extended spectrum beta lactamase (ESBL) producing Gram-negative bacilli (GNB) ${ }^{8,9}$ Most studies showed gram negative bacilli among the most frequently isolated MDR bacteria in severe COPD exacerbations. This included Pseudomonas aeruginosa, Acinetobacter baumanii and Stenotrophomonas maltophilia. ${ }^{8}$

In this study, bacterial culture and drug susceptibility of induced sputum samples were done in severe and very severe COPD patients to identify the sensitivity pattern in infective exacerbations of COPD.

\section{METHODS}

Study population: The patients with COPD on standard optimal medical therapy were selected as the study population from Shree Birendra Hospital, Chhauni, Kathmandu. Total of 89 subjects with positive sputum culture for clinically significant bacterial pathogens were included. All the patients were admitted to the hospital due to AE. AE of COPD was defined according to the guidelines of Global Initiative for Obstructive Lung Disease (GOLD). ${ }^{10}$ The subjects with two or more exacerbations of COPD in a year and frequent use of antibiotics were included in the study. The sputum samples were collected for the study between July 2013 and June 2014.

Sputum sample collection: Sputum samples were collected from all patients on day one of presentation before first dose of antibiotics in emergency department. All subjects rinsed mouth with sterile water which was followed by nebulisation with Salbutamol. The expectorated sputum was visually inspected for adequacy and was submitted to the microbiology lab within two hours ${ }^{11}$.

Sample processing: Sputum samples were processed and identified according to guidelines given by American Society of Microbiology. Sensitivity was carried out by Kirby Bauer's method as per Clinical and Laboratory Standards Institute (CLSI) recommendations. Due to extensive variation in definition of MDR, this study adopted interim standards provided for acquired multidrug resistance by European Centre for Disease Control and Prevention. Each organism was labelled MDR as per the guidelines stated in the literature ${ }^{12,13 .}$

Statistical analysis: Statistical analysis was done with IBM SPSS version 20. All the categorical data were expressed in percentage and absolute numbers. The continuous numerical data were expressed as mean \pm Standard deviation. The statistical significance was set at $\mathrm{p}<0.05$ with $95 \%$ confidence. The statistical evaluation for categorical variables were done using chi square test.

\section{RESULTS}

The eighty nine positive sputum cultures were included in the study. Isolated bacterial pathogens were tested for relevant antibiotics as per recommendations for their drug 
Table 1: The organisms isolated in the bacterial cultures of expectorated sputum samples. Total samples: 89 .

\begin{tabular}{|l|c|c|}
\hline Organisms & Number(n) & Percent \\
\hline Gram Negative & $\mathbf{7 9}$ & $\mathbf{8 8 . 7 6 \%}$ \\
\hline $\begin{array}{l}\text { Pseudomonas } \\
\text { aeruginosa }\end{array}$ & 26 & 29.22 \\
\hline Acinetobacter sp. & 18 & 20.22 \\
\hline Klebsiella Sp. & 18 & 20.22 \\
\hline $\begin{array}{l}\text { Klebsiella } \\
\text { pneumonia }\end{array}$ & 15 & - \\
\hline Klebsiella oxytoca & 3 & - \\
\hline Escherichia coli & 11 & 12.36 \\
\hline Citrobacterfreundii & 4 & 4.49 \\
\hline $\begin{array}{l}\text { Moraxella } \\
\text { catarrhalis }\end{array}$ & 2 & 2.25 \\
\hline Gram Positive & $\mathbf{1 0}$ & $\mathbf{1 1 . 2 4 \%}$ \\
\hline Streptococcus sp. & 6 & 6.75 \\
\hline Staphylococcus & 4 & 4.49 \\
\hline $\begin{array}{l}\text { MRSA } \\
\text { MSSA }\end{array}$ & 3 & - \\
\hline sensivity. & 1 & - \\
\hline
\end{tabular}

sensitivity. The isolated pathogens in sputum cultures are enlisted in table no. 1.

\section{Sensitivity and Resistance of Organisms}

Pseudomonas aeruginosa: was the most commonly isolated organism in our study. Among the penicillin group of antibiotics all growths of Pseudomonas sp. were found resistant to Amoxicillin (4 out of 4) and Coamoxyclav (9 out of 9). Eighteen out of 21 ( $85.71 \%$ of 21 ) isolated Pseudomonas sp. were sensitive to PTZ. Only 3 out of 21 (14.28\% of 21) were found to resistant to PTZ. Among the quinolones $83.33 \%$ (20 of 24) isolates were sensitive and $16.66 \%$ (4 of 24) were resistant to Ciprofloxacin whereas $71.42 \%$ (5 of 7) MJSBH Vol 15 Issue 1 Jan- Jun 2016 isolates were sensitive and $28.57 \%$ ( 2 of 7 ) were resistant to Ofloxacin. All growths tested with Co-trimoxazole were resistant to it (4 of 4). Twelve growths tested for Chloramphenicol sensitivity showed sensitive strains in seven $(58.3 \%, 7$ of 12$)$ and resistant strains in five ( $41.67 \%, 5$ of 12 ). Among the aminoglycosides Amikacin was sensitive in $91.30 \%$ (21 of 23 ) isolates and resistant in two isolates ( $8.69 \%, 2$ of 23 ) whereas Gentamycin was sensitive in $84.21 \%$ (16 of 19) isolates and resistant in $15.78 \%$ (3 of 19 ). Tobramycin was tested in twoisolates and both were sensitive. Doxycycline sensitivity was tested in 22 culture isolates where $40.90 \%$ (9 of 22) were sensitive and $59.09 \%$ (13 of 22) were resistant. Cephalexin resistance was shown by all tested isolates (2 of 2), Ceftriaxone resistance was shown by $66.66 \%$ isolates (4 of 6 ) and sensitivity by $33.33 \%$ ( 2 of 6 ), Cefotaxime resistance was shown by $83.33 \%$ isolates ( 5 of 6) and sensitivity by $16.67 \%$ ( 1 of 6 ). All 24 culture growths showing pseudomonas were tested for Ceftazidime sensitivity. 69.23\% (18 of 26) were sensitive and $30.70 \%$ ( 8 of 26 ) were resistant. All samples tested for Cefixime sensitivity were found resistant (2 of 2). Cefepime sensitivity was shown by $57.89 \%$ isolates (11 of 19) and resistance by $42.11 \%$ (8 of 19). All isolates tested for Imipenem sensitivity were found sensitive (9 of 9) whereas $88.89 \%$ were sensitive to Meropenem ( 8 of 9) and $11.11 \%$ (1 of 9) were resistant. All isolates tested for Carbenicillin (6 of 6), Colistin (9 of 9) and Polymyxin B (7 of 7) were found sensitive to respective drugs. Pseudomonas sp. showing resistance to two or more drugs of different mechanism of actions (MDR) were $62.5 \%$ (15 of 24$)$. 
Table 2: Gram Negative Bacilli isolated from the COPD subjects during study period are listed along the columns. The rows enumerate the antibiotics tested for individual organisms. The highlighted cells show number of samples in denominators. $\mathrm{S}=$ Sensitive and $\mathrm{R}=$ Resistant.

\begin{tabular}{|c|c|c|c|c|c|c|c|}
\hline \multirow[b]{2}{*}{ Antibiotics } & \multicolumn{2}{|c|}{ Gram Negative Bacilli } & \multirow[b]{2}{*}{ Klebsiella oxytoca } & \multirow[b]{2}{*}{ E coli } & \multirow[b]{2}{*}{ Acine tobacter Sp } & \multirow[b]{2}{*}{ Citrobacter Sp } & \multirow[b]{2}{*}{ Moraxella Cattarhalis } \\
\hline & Pseudomonas & Klebsiella pne umoniae & & & & & \\
\hline Ampicillin & Not Tested & Not Tested & Not tested & Not tested & Not tested & Not tested & Not tested \\
\hline Amoxycillin & $R-4 / 4$ & S $1 / 4$ R $3 / 4$ & R $1 / 1$ & S $1 / 4$ R $3 / 4$ & R $9 / 9$ & S $1 / 4$ R $3 / 4$ & Not tested \\
\hline Amoxyclav & R-9/9 & S $1 / 10$ R $9 / 10$ & R $1 / 1$ & $\mathrm{R} 8 / 8$ & $\mathrm{R} 12 / 12$ & $\mathrm{~S} 1 / 4 \mathrm{R} 3 / 4$ & Not tested \\
\hline cloxacillin & $\mathrm{S}-2 / 2$ & Not Tested & Not tested & $\mathrm{R} 1 / 1$ & $\mathrm{R} 1 / 1$ & Not tested & Not tested \\
\hline PTZ & S-18/21 R-3/21 & $\mathrm{S} 4 / 10 \mathrm{R} 6 / 10$ & $\mathrm{~S} 1 / 1$ & S 3/7 R 4/7 & S $3 / 15 \mathrm{R} 12 / 15$ & $\mathrm{~S} 1 / 1$ & Not tested \\
\hline Nalidixic acid & Not Tested & Not Tested & Not tested & Not tested & Not tested & Not tested & Not tested \\
\hline Norfloxacin & Not Tested & Not Tested & Not tested & Not tested & Not tested & Not tested & Not tested \\
\hline Ciprofloxacin & S-20/24 R 4/24 & S 7/13 R 6/13 & $\mathrm{S} 2 / 2$ & S 4/9 R 5/9 & S $2 / 14 \mathrm{R} 12 / 14$ & S $1 / 4$ R $3 / 4$ & $\mathrm{~S} 2 / 2$ \\
\hline Ofloxacin & S $5 / 7$ R $2 / 7$ & S $3 / 9$ R $6 / 9$ & S $1 / 1$ & S $3 / 7$ R $4 / 7$ & S $2 / 10 \mathrm{R} 8 / 10$ & R $1 / 1$ & $\mathrm{~S} 2 / 2$ \\
\hline Nitrofurantoin & Not Tested & Not Tested & Not tested & Not tested & Not tested & Not tested & Not tested \\
\hline Cotrimoxazole & R $4 / 4$ & S $4 / 8 \mathrm{R} 4 / 8$ & $\mathrm{~S} 1 / 1$ & S $4 / 6$ R $2 / 6$ & S $1 / 6$ R $5 / 6$ & S $3 / 4$ R $1 / 4$ & $\mathrm{R} 2 / 2$ \\
\hline Chloramphenico & IS $7 / 12$ R 5/12 & S $7 / 10$ R $3 / 10$ & $\mathrm{~S} 2 / 2$ & S $7 / 8$ R $1 / 8$ & S $4 / 9$ R $5 / 9$ & S $3 / 4$ R $1 / 4$ & $\mathrm{~S} 2 / 2$ \\
\hline Erythromycin & Not Tested & Not Tested & Not tested & Not tested & Not tested & Not tested & $\mathrm{S} 2 / 2$ \\
\hline Azithromycin & Not Tested & Not Tested & Not tested & Not tested & Not tested & Not tested & $\mathrm{S} 2 / 2$ \\
\hline Amikacin & $\mathrm{S} 21 / 23 \mathrm{R} 2 / 23$ & S 8/13 R 5/13 & S $1 / 1$ & S $7 / 8$ R $1 / 8$ & S $3 / 15$ R $12 / 15$ & S $3 / 4$ R $1 / 4$ & Not tested \\
\hline Gentamicin & S $16 / 19$ R 3/19 & S 6/11 R 5/11 & S $1 / 1$ & S $5 / 8$ R $3 / 8$ & S 4/11 R 7/11 & S $4 / 4$ & Not tested \\
\hline Tobramycin & $\mathrm{S} 2 / 2$ & Not Tested & Not tested & Not tested & Not tested & Not tested & Not tested \\
\hline Tetracycline & Not Tested & Not Tested & Not tested & Not tested & Not tested & Not tested & Not tested \\
\hline Doxycycline & $\mathrm{S} 9 / 22 \mathrm{R} 13 / 22$ & S 9/12 R 3/12 & $\mathrm{S} 2 / 2$ & $\mathrm{~S} 4 / 8 \mathrm{R} 4 / 8$ & S 4/13 R 9/13 & $\mathrm{S} 4 / 5 \mathrm{R} 1 / 5$ & Not tested \\
\hline Cefalaxin & R $2 / 2$ & S 2/7 R 5/7 & Not tested & $\mathrm{S} 2 / 4 \mathrm{R} 2 / 4$ & R $3 / 3$ & Not tested & Not tested \\
\hline Ceftriaxone & $\mathrm{S} 2 / 6 \mathrm{R} 4 / 6$ & S 6/11 R 5/11 & S $1 / 1$ & S 4/7 R 3/7 & S $2 / 9$ R $7 / 9$ & S $3 / 4$ R $1 / 4$ & $\mathrm{~S} 2 / 2$ \\
\hline Cefotaxim & S $1 / 6$ R $5 / 6$ & S 5/12 R 7/12 & $\mathrm{S} 2 / 2$ & S $4 / 4$ R $4 / 4$ & S $1 / 10$ R $9 / 10$ & $\mathrm{~S} 3 / 4 \mathrm{R} 1 / 4$ & $\mathrm{R} 2 / 2$ \\
\hline Cefepime & S 11/19 R 8/19 & S 6/12 R 6/12 & $\mathrm{S} 1 / 1$ & S $2 / 8 \mathrm{R} 6 / 8$ & S $1 / 11$ R $10 / 11$ & S $5 / 5$ & Not tested \\
\hline Ceftazidime & S $18 / 26$ R $8 / 26$ & $S 4 / 10$ R $6 / 10$ & $\mathrm{~S} 1 / 1$ & S $4 / 4$ R $4 / 4$ & R 11/11 & $\mathrm{S} 4 / 4$ & Not tested \\
\hline Cefixime & R $2 / 2$ & R $1 / 1$ & Not tested & Not tested & Not tested & Not tested & Not tested \\
\hline Vancomycin & Not Tested & Not Tested & Not tested & Not tested & Not tested & Not tested & Not tested \\
\hline Imipenem & S 9/9 & $\mathrm{S} 1 / 1$ & Not tested & $\mathrm{S} 2 / 2$ & S $5 / 5$ & Not tested & Not tested \\
\hline Meropenam & S $8 / 9 \mathrm{R} 1 / 9$ & $\mathrm{~S} 2 / 3 \mathrm{R} 1 / 3$ & Not tested & S $3 / 4$ R $1 / 4$ & S $3 / 7$ R $4 / 7$ & S $3 / 3$ & $\mathrm{~S} 2 / 2$ \\
\hline Polymyxin B & S $7 / 7$ & Not Tested & Not tested & Not tested & $\mathrm{S} 1 / 1$ & Not tested & Not tested \\
\hline Colistin & S $9 / 9$ & Not Tested & Not tested & Not tested & $\mathrm{S} 1 / 1$ & Not tested & Not tested \\
\hline Clindamycin & Not Tested & Not Tested & Not tested & Not tested & Not tested & Not tested & Not tested \\
\hline Carbenicllin & S $6 / 6$ & Not Tested & Not tested & Not tested & Not tested & Not tested & Not tested \\
\hline Levoflox & Not Tested & Not Tested & Not tested & Not tested & Not tested & Not tested & Not tested \\
\hline
\end{tabular}

Acinetobacter sp.: was predominantly resistant to pencillins: Amoxycillin $100 \%$ (9 of 9), Amoxyclav $100 \%$ (12 of 12), PTZ $80 \%$ (12 of 15). Quinolones also had low sensitivity: Ciprofloxacin was resistant in $85.71 \%$ (12 of 14) and sensitive in $14.29 \%$ (2 of 14$)$. Ofloxacin was resistant in $80 \%$ (8 of 10) and sensitive in $20 \%$ (2 of 10). Co-trimoxazole was resistant in $83.33 \%$ (5 of 6 ) and sensitive in $16.67 \%$ ( 1 of 6 ) whereas Chloramphenicol was sensitive in $44.44 \%$ (4 of 9) and resistant in $55.55 \%$ (5 of 9). Amikacin sensitivity was $20 \%$ (3 of 15 ) and resistance $80 \%$ (12 of 15) whereas Gentamycin sensitivity was $36.36 \%$ (4 of 11) and resistance was $63.64 \%$ (7 of 11). Doxycycline sensitivity was $30.77 \%$ (4 of 13) and resistance was $69.23 \%$ (9 of 13 ).
Cephalexin was resistant in all tested isolates (3 of 3 ).

Ceftriaxone resistance was $77.78 \%$ (7 of 9) and sensitivity was $22.22 \%$ ( 2 of 9). Ninety percent (9 of 10) isolates showed resistance to Cefotaxime and sensitivity was $10 \%$. Cefepime resistance was $90.9 \%$ (10 of 11) with sensitivity of $9.1 \%$ ( 1 of 11$)$. All isolates tested for Ceftazidime sensitivity showed resistance $(100 \%, 11$ of 11$)$. On the contrary, all isolates tested for Imipenem sensitivity showed sensitive results $(100 \%, 5$ of 5$)$ whereas $42.85 \%$ (3 of 7 ) showed sensitivity to Meropenem and $57.15 \%$ (4 of 7) showed resistance. Colistin and Polymyxin B were tested in one culture growth each showing sensitivity. Thirteen out of 16 Acinetobacter isolates $(81.25 \%)$ showed resistance to two or 
more drugs of different mechanism of action (MDR).

Klebsiella: was isolated in total 15 samples. 13 samples were Klebsiella pneumoniae and 2 samples were Klebsiella oxytoca. The isolated species of Klebsiella were drug sensitive NonMDR pathogens in eight samples. Whereas MDR pathogens were detected in seven instance. Among the B-lactam group of antibiotics, organisms were found resistant to amoxicillin in 4 out of 5 samples ( $80 \%)$, to Co-amoxyclav in 10 out of 11 samples (90.9 $\%)$ and to PTZ in 6 out of 11 samples (54.54 $\%)$. When Cephalosporins were evaluated Cephalexin was resistant in 5 out of 7 samples $(71.42 \%)$, Ceftriaxone was resistant in 5 out of 12 samples (41.67\%), Cefotaxime was resistant in 7 out of 14 samples (50\%), Cefepime was resistant in 6 out of 13 samples (46.15\%) and Ceftazidime was resistant to 6 out of 11 samples (54.54\%). Among the Quinolones Klebsiella was found sensitive to Ciprofloxacin in 9 out of 15 samples (60\%) and to Ofloxacin in 4 out 10samples (40 $\%)$.When aminoglycosides were evaluated Amikacin was found sensitive in 9 out of 14 samples $(64.28 \%)$ and Gentamicin was found sensitive in 7 out of 12 samples (58.33\%). Among tetracyclines only Doxycycline was evaluated which showed sensitive results in 11 out of 14 samples $(78.57 \%)$. Chloramphenicol was found sensitive in 9 out of 12 samples ( 75 $\%$ whereas Co-trimoxazole was found sensitive in 5 out of 9 samples (55.56\%). Carbapenem was tested in only three suptum samples. Meropenem was sensitive in 2 out of 3 samples (66.66 \%) whereas Imipenem was sensitive in all three samples (100\%).

E. coli: Total 11 sputum cultures showed E.coli. Among these 6 isolates were MDR pathogens $(54.54 \%)$ and five isolates were sensitive organisms (45.45 \%). Among Penicillin groups: Amoxicillin was resistant in 3 out 4 samples (75\%), Co-amoxyclav was resistant in 8 out of 8 samples $(100 \%)$ and PTZ was resistant in 4 out of 7 samples (57.14 $\%)$. When Cephalosporins were evaluated Cephalexin was resistant in 2 out of 4 samples (50\%), Ceftriaxone was resistant in 3 out of 7 samples (42.85\%), Cefotaxime was resistant in 4 out of 8 samples (50\%), Cefepime was resistant in 6 out of 8 samples $(75 \%)$ and Ceftazidime was resistant in 4 out of 8 samples (50 \%). Among the Quinolones E.coli was found sensitive to Ciprofloxacin in 4 out of 9 samples $(44.44 \%)$ and to Ofloxacin in 3 out 7 samples (42.85\%).When aminoglycosides were evaluated Amikacin was found sensitive in 7 out of 8 samples $(87.5 \%)$ and Gentamicin was found sensitive in 5 out of 8 samples $(62.50 \%)$. Among tetracyclines only Doxycycline was evaluated which showed sensitive results in 4 out of 8 samples ( $50 \%$ ). Chloramphenicol was found sensitive in 7 out of 8 samples $(87.5 \%$ ) whereas Co-trimoxazole was found sensitive in 4 out of 6 samples (66.67 \%). Carbapenem was tested in only four sputum samples. Meropenem was sensitive in 3 out of 4 samples $(75 \%)$ whereas Imipenem was sensitive in all two samples (100\%).

Citrobacter sp.: There were four isolates of Citrobacter freundii. Two of these were MDR pathogens (50\%). Amoxicillin was resistant in $75 \%$ samples (3 out of 4), Co-amoxyclav in 75 $\%$ samples (3 out of 4 ) and PTZ in $100 \%$ (1 out of 1). Cephalosporins were evaluated showing both Ceftriaxone and Cefotaxime resistance in 1 out of 4 samples (25\%). Cefepime and Ceftazidime were sensitive in all four samples (100\%).Among the Quinolones, 
Citrobacter was found sensitive to Ciprofloxacin in 3 out of 4 samples $(75 \%)$ and resistant to Ofloxacin in 1 out 1 sample (100 $\%)$.When aminoglycosides were evaluated Amikacin was found sensitive in 3 out of 4 samples $(75 \%)$ and Gentamicin was found sensitive in all $4(100 \%)$.Among tetracycline only Doxycycline was evaluated which showed sensitive results in 3 out of 4 samples (75\%). Chloramphenicol and Co-trimoxazole were found sensitive in 3 out of 4 samples (75\%). Among Carbapenem only Meropenem was tested which was sensitive in all 3 samples $(100 \%)$.

Moraxella catarrahalis: Two isolates of Moraxella catarrahalis were both sensitive to Ciprofloxacin, Ofloxacin, Chloramphenicol, Azithromycin, Ceftriaxone and Meropenem. But these isolates were resistant to Cotrimoxazole and Cefotaxim.

Gram Positive Organisms: Six isolates of Streptococcus were resistant to Amoxicillin, Co-amoxyclav, Erythromycin and Gentamicin but were sensitive to Chloramphenicol and Vancomycin. Among the four isolates of Staphylococcus three were MRSA and one MSSA. MRSA was resistant to Ampicillin, Amoxicillin, Co-amoxyclav, Ofloxacin, Doxycycline and Ceftriaxone. The overall susceptibility pattern of individual antibiotics is given in figure no. 6 .

\section{DISCUSSION}

The most commonly isolated community acquired organisms in AE COPD are Haemophilus influenzae, Streptococcus pneumoniae and Moraxella catarrhalis on lower respiratory tract samples. ${ }^{14}$ Our study showed very few isolates of community acquired pathogens in COPDs. On the contrary,
Pseudomonas sp., Klebsiella sp., Acinetobacter $s p$. and Enterobacter sp. were commonly isolated from $\mathrm{AE}$ COPD patients requiring intensive care and mechanical ventilations in an article published from Thailand in 2012. ${ }^{15}$ In accordance to this our study also isolated GNBs as the most common organisms in COPD subjects during AE. Our study isolated significantly larger number of GNBs as compared to GPCs, which is in accord to other South East Asian Studies. ${ }^{16,17}$

Though viruses are the most common pathogens for $\mathrm{AE}$ of $\mathrm{COPD}^{17}$, due to the lack of virology facility in our institute we were not able to conduct virological isolations. Hence our study prospectively limited the protocol to bacterial cultures. Pseudomonas aeruginosa, Acinetobacter baumannii, Klebsiella pneumonia and E. coli (in descending order) were the most common GNBs isolated in severe and very severe COPD patients in our study. Similar sequence of pathogens were identified by a study conducted by Dai M.Y. et al on AE of COPD in People's Republic of China. ${ }^{17}$

Incidence of MDR pathogens occurring in COPD patients is also well documented. Frequent exacerbations and frequent visits to hospital for treatment with repeated use of antibiotics tend to play significant role in involvement of MDR GNBs in AE of COPD. In severe COPD Pseudomonas aeruginosa, Acinetobacter baumannii and Klebsiella pneumoniae are often MDR. ${ }^{18}$ Resistance to two or more class of drugs was observed in all these organisms in high percentage in our study. This probably represented the hospital acquired pathogens with repeated visits and frequent use of antibiotics in this study population. 
Pseudomonas $s p$. was completely resistant to Amoxicillin, Co-amoxiclav, Cotrimoxazole, Cephalexin and Cefixime. Good sensitivity was identified for PTZ, Quinolones and aminoglycosides. Carbapenems, Polymyxin B and Colistin were the most effective antibiotics. Similar resistance pattern was also observed in a large multicentre Chinese study ${ }^{19}$ where Amoxicillin, Co-amoxiclav and Cotrimoxazole were the most ineffective drugs against Pseudomonas sp. In their study Amikacin and Cefoperazone faired best but in our study Amikacin and Carbapenems were the best agents. Imipenem resistance for Pseudomonas was very high in the Chinese study. On the contrary, Imipenem was the most effective drug in our study population for all the GNBs.

Most of the other GNB in our study showed similar sensitivity pattern with Penicillin groups being the most ineffective and Carbapenems being the most effective drugs. However Aminoglycosides and Quinolones had good sensitivity for GNBs as the first line agents.

On the contrary, Acinetobacter sp. showed worst resistance pattern, with penicillins, PTZ, Quinolones, Co-trimoxazole, Aminoglycosides and Cephalosporins being largely ineffective. High occurrence of MDR Acinetobacter (81.25 $\%)$ might represent circulation from a common hospital source. In a retrospective study on occurrence of Acinetobacter sp., $79.5 \%$ initial isolates were MDR pathogens in a single health institute, which was similar to our study 20 . Afore mentioned study also highlighted COPD as the risk factor for mortality with Acinetobacter infection. Carbapenem resistance among MDR Acinetobacter circulating in institutes have been well documented. ${ }^{20}$ However Imipenem was the most effective antibiotic in our study. Colistin and polymyxin B were tested on limited MDR samples and were also found sensitive.

Very few GPCs were isolated in our study. Considering the study population with chronic airway disease with repeated hospital visits and repeated use of antibiotics, predominant growth of GNBs are explainable.16-19 Laboratory limitations on the growth of GPCs, if at all they exist, are unexplainable. And the transportation time and processing time has been strictly followed during the study period.

During the study period a very good sensitivity to Carbapenems were observed particularly Imipenem. Such relative sparing of a group of antibiotics could be attributed to limited use of the agent under proper authorization during the study period in the institute. However, sensitivity has gradually declined to Carbapenems with their relative ease of availability after this study period. Colistin and Polymyxin B still have good sensitivity with its less frequent availability in the institute.

CONCLUSION: Gram negative bacteria are more commonly isolated in AE of COPD as compared to Gram positives. Pseudomonas aeruginosa, Acinetobacter baumannii, Klebsiella pneumonia and E. Coli are the most common Gram negatives isolated. The multi drug resistant gram negatives are common in COPDs with frequent exacerbations, antibiotic use and hospital visits. Aminoglycosides and Quinolones have good sensitivity to GNBs. However, Carbapenems were found to be the most effective agents against these organisms. 


\section{REFERENCES}

1. Who.int. World Health Organisation. Chronic obstructive pulmonary disease (COPD) [Internet]. 2015 [cited 2 June 2015]. Available from: http://www.who.int/ mediacentre/factsheets/fs315/en/

2. Burge S, Wedzicha JA. COPD exacerbations: definitions and classifications. Eur Respir J 2003; 21: Suppl. 41, 46s-53s. DOI: http://dx.doi.org/ 10.1183/09031936.03.00078002

3. Sethi S, Jones PW, Theron MS, Miravitlles M, Rubinstein E, Wedzicha JA, et al. Pulsed moxifloxacin for the prevention of exacerbations of chronic obstructive pulmonary disease: a randomized controlled trial. Respiratory Research 2010, 11:10.

http://dx.doi.org/10.1186/1465-9921-11-10 PMid:20109213

4. Lin SH, Kuo PH, Hsueh PR, Yang PC, Kuo SH. Sputum bacteriology in hospitalized patients with acute exacerbation of chronic obstructive pulmonary disease in Taiwan with an emphasis on Klebsiellapneumoniae and Pseudomonas aeruginosa. Respirology. 2007 Jan; 12(1):81-7. http://dx.doi.org/ 10.1111/j.1440-1843.2006.00999.x PMid: 17207030

5. Pela R, Marchesani F, Agostinelli C, Staccioli D, Cecarini L, Bassotti C, Sanguinetti CM. Airways microbial flora in COPD patients in stable clinical conditions and during exacerbations: a bronchoscopic investigation. Monaldi Arch Chest Dis. 1998 Jun;53(3):262-7. PMid:9785808

6. Domenech A, Puig C, Marti S, Santos S, Fernandez A, Calatayud L, et al. Infectious etiology of acute exacerbations in severe COPD patients. J Infect. 2013 Dec;67(6): MJSBH Vol 15 Issue 1 Jan- Jun 2016 516-23.http://dx.doi.org/10.1016/j.jinf. 2013.09.003 PMid:24055804

7. Ye F, He LX, Cai BQ, Wen FQ, Chen BY, Hadiarto $M$, et al. Spectrum and antimicrobial resistance of common pathogenic bacteria isolated from patients with acute exacerbation of chronic obstructive pulmonary disease in mainland of China. Chin Med J (Engl). 2013 Jun; 126(12):2207-14.

8. Nseir S, Ader F. Prevalence and Outcome of Severe Chronic Obstructive Pulmonary Disease Exacerbations Caused by Multidrug-resistant Bacteria. CurrOpinPulm Med. 2008;14(2):95-100. http://dx.doi.org/ $\underline{10.1097 / \mathrm{mcp} .0 \mathrm{~b} 013 \mathrm{e} 3282 \mathrm{f3} 7 \mathrm{a} 11}$

9. Nseir S, Di Pompeo C, Cavestri B, JozefowiczE,Nyunga $\mathrm{M}$, Soubrier $\mathrm{S}$, et al. Multiple-drug-resistant bacteria in patients with severe acute exacerbation of chronic obstructive pulmonary disease: Prevalence, risk factors, and outcome. Crit Care Med. 2006 Dec; 34(12):2959-66. http:// dx.doi.org/10.1097/01.CCM. 0000245666.28867.C6 PMid:17012911

10. Global Strategy for the Diagnosis, Management and Prevention of COPD, Global Initiative for Chronic Obstructive Lung Disease (GOLD) [Internet]. 2015 [cited 22 June 2015]. Available from: http:// www.goldcopd.org/guidelines-globalstrategy-for-diagnosis-management.html

11. Gupta KB, Garg S. Sputum induction- A useful tool in respiratory diseases. Lung India 2006;23:82-6. http://dx.doi.org/ 10.4103/0970-2113.44416

12. Magiorakos AP, Srinivasan A, Carey RB, Carmeli Y, Falagas ME, Giske CG, et al. Multidrug-resistant, extensively drug- 
resistant and pandrug-resistant bacteria: an international expert proposal for interim standard definitions for acquired resistance. ClinMicrobiol Infect. 2012 Mar;18(3): 268-81. http://dx.doi.org/10.1111/j. 1469-0691.2011.03570.x PMid:21793988

13. Clinical and Laboratory Standards Institute. Performance standards for antimicrobial susceptibility testing. 23rd informational supplement. Wayne PA. CLSI 2013; M100S23.

14. Soler N, Torres A, Ewig S,Gonzalez J, Celis R, El-Ebiary $\mathrm{M}$, et al. Bronchial microbial patterns in severe exacerbations of chronic obstructive pulmonary disease (COPD) requiring mechanical ventilation. Am J Respir Crit Care Med. 157 (5 Pt 1) (1998), pp. 1498-505. http://dx.doi.org/ 10.1164/ajrccm.157.5.9711044 PMid: 9603129

15. Siripataravanit S, Phaicharoen R, Termsetcharoen S, Klangprapun N. Bacteria associated with acute exacerbations of chronic obstructive pulmonary disease requiring mechanical ventilation and antimicrobial management in Respiratory Care Unit of Central Chest Institute of Thailand. J Med Assoc Thai, 95 (Suppl 8 (August)) (2012), pp. 11-18.

16. Sharan H. Aerobic Bacteriological Study of Acute Exacerbations of Chronic Obstructive Pulmonary Disease.J Clin Diagn Res.2015 Aug;9(8):DC10-2. doi: 10.7860/JCDR/ 2015/14515.6367. http://dx.doi.org/10.7860/ JCDR/2015/14515.6367
17. Dai MY, Qiao JP, Xu YH, Fei GH. Respiratory infectious phenotypes in acute exacerbation of COPD: an aid to length of stay and COPD Assessment Test.Int J Chron Obstruct Pulmon Dis. 2015 Oct 20;10:2257-63. doi: 10.2147/COPD.S92160 http://dx.doi.org/10.2147/COPD.S92160

18. Talbot GH, Bradley J, Edwards Jr. JE, Gilbert D, Scheld M, Bartlett JG. Bad bugs need drugs: an update on the development pipeline from the Antimicrobial Availability Task Force of the Infectious Diseases Society of America. Clin Infect Dis, 2006;42:657-68.

http://dx.doi.org/10.1086/499819

PMid:16447111

19. Ma X, Cui J, Wang J, Chang Y, Fang Q, Bai $\mathrm{C}$ et al. Multicentre investigation of pathogenic bacteria and antibiotic resistance genes in Chinese patients with acute exacerbation of chronic obstructive pulmonary disease.J Int Med Res. 2015 Oct; $43(5): 699-710$. d o i : $10.1177 / 0300060515587577$.

20. Townsend J, Park AN, Gander R, Orr K, Arocha D, Zhang S, et al. Acinetobacter infections and outcomes at an academic medical center: a disease of long-term care. Open Forum Infect Dis. 2015;2(1):ofv023. doi: 10.1093/ofid/ofv023. 